\title{
Engineering Yarrowia lipolytica for arachidonic acid production through rapid assembly of metabolic pathway
}

Hu-Hu Liu ${ }^{1}$, Catherine Madzak $^{2}$, Mei-Li Sun ${ }^{1}$, Lu-Jing Ren ${ }^{1,5}$, Ping Song ${ }^{1,5}$, He Huang ${ }^{3,4,5}$,

$$
\text { Xiao-Jun } \mathrm{Ji}^{1,5^{*}}
$$

1. College of Biotechnology and Pharmaceutical Engineering, Nanjing Tech University, No. 30 South Puzhu Road, Nanjing 211816, People's Republic of China

2. GMPA, AgroParisTech, INRA, Université Paris-Saclay, 78850, Thiverval-Grignon, France

3. School of Pharmaceutical Sciences, Nanjing Tech University, No. 30 South Puzhu Road, Nanjing 211816, People's Republic of China

4. State Key Laboratory of Materials-Oriented Chemical Engineering, Nanjing Tech University, No.5 Xinmofan Road, Nanjing 210009, People’s Republic of China

5. Jiangsu National Synergetic Innovation Center for Advanced Materials (SICAM), No.5 Xinmofan Road, Nanjing 210009, People’s Republic of China

*Corresponding authors: Xiao-Jun Ji (E-mail: xiaojunji@njtech.edu.cn, Tel: +86 25 58139942;

Fax: +86 25 58139389) 


\section{Abstract}

Yarrowia lipolytica, a non-conventional oleaginous yeast with special traits, has attracted increasing interest for producing value-added products. Generally, the DNA fragments of these heterologous metabolic pathways are constructed via the classic restriction digestion and ligation method. In contrast, the one-step in vivo pathway assembly method has been only rarely applied to $Y$. lipolytica. Here, with arachidonic acid biosynthesis as a case study, a one-step in vivo pathway assembly and integration method was used for engineering $Y$. lipolytica. Using rDNA as integrative locus, this study showed that there was a relation between the assembly efficiency and the length of overlapping region. Especially, with an overlap up to $1 \mathrm{~kb}$, the method was able to rapidly assemble the arachidonic acid biosynthesis pathway (nearly $10 \mathrm{~kb}$ ) into the chromosome with high efficiency (nearly 23\%). Meanwhile, the pathway assembled in $Y$. lipolytica demonstrated long-term genetic stability and the engineered strain exhibited robust growth. Furthermore, this study demonstrated that the codon-optimized genes from Mortierella alpina can function efficiently in $Y$. lipolytica: a high level arachidonic acid production ( $0.4 \%$ of total fatty acids) was produced in the engineered strain. To our knowledge, this is the first time that this method is applied to $Y$. lipolytica for functional polyunsaturated fatty acids production. This method represents a powerful tool with potential for facilitating engineering applications in non-conventional yeasts.

\section{Keywords:}

Yarrowia lipolytica, arachidonic acid, in vivo, pathway assembly, synthetic biology 


\section{Introduction}

With the development of metabolic engineering and synthetic biology, the microbial-based biotechnology has been a promising strategy for production of value-added chemicals in heterologous microorganisms [1-2]. Generally, these biochemical pathways are constructed in conventional microorganisms such as Escherichia coli and Saccharomyces cerevisiae [3-4]. Meanwhile, non-conventional yeasts with special traits are viewed as important resources and have received growing attention [5]. Among them, especially, Yarrowia lipolytica is able to assimilate and ferment various carbon substrates and produce recombinant proteins as well as various chemicals, such as organic acids, etc [6-10]. Recently, with the development of yeast biotechnology, this non-conventional yeast has been used as a platform factory for production of various novel value-added chemicals, such as lycopene, eicosapentaenoic acid and fatty alcohol [11-14].

Interestingly, functional polyunsaturated fatty acids (PUFAs), including omega-6 and omega-3, have been proved to play important functions on human health. Especially, arachidonic acid (ARA), a typical omega-6 PUFA, is synthesized via the aerobic $\Delta-6$ desaturation and elongation pathway in the fungus Mortierella alpina [15]. Meanwhile, based on the assembly of the desaturation and elongation pathway, ARA is also produced in various organisms, such as Arabidopsis thaliana, Brassica napus and S. cerevisiae [16-17]. The ARA biosynthesis pathway in these engineered chassis was constructed via the classic restriction digestion and ligation method. However, with the synthetic biology development, various in vitro and in vivo DNA assembly 
methods are now available for constructing metabolic pathways [18]. Generally, these developed pathway assembly and integration methods, such as ePathBrick, PADOM, LCR assembly and DNA assembler, are mainly applied to E. coli and S. cerevisiae [19-20]. Compared with the classic restriction digestion and ligation method, the in vivo one-step pathway assembly allows for the reduction of the time and cost for constructing the biochemical pathway. However, such in vivo one-step pathway assembly methods have been rarely applied to the non-conventional yeast $Y$. lipolytica [21], and never to our knowledge for functional PUFAs production.

In this study, to demonstrate the possibility and efficiency of pathway assembly in the non-conventional yeast $Y$. lipolytica, we used arachidonic acid biosynthesis as a case study. The corresponding aerobic $\Delta-6$ desaturation and elongation pathway was designed and constructed via one-step in vivo pathway assembly. Meanwhile, the relationship between the assembly efficiency and the length of overlapping region was demonstrated. Furthermore, analyses were performed throughout the time course of the fermentation in order to assess the effect of the heterologous metabolic pathway in this non-conventional yeast. It was demonstrated that the codon-optimized genes from Mortierella alpina can function in $Y$. lipolytica. Altogether, the engineered $Y$. lipolytica strain was demonstrated to be a potential arachidonic acid producer with long-term genetic stability and robust growth. This study shows that the in vivo one-step pathway assembly and integration method is capable of facilitating the engineering of $Y$. lipolytica for biotechnological applications.

\section{Materials and methods}




\subsection{Microbial strains, media and culture conditions}

E. coli DH5 $\alpha$ was used for cloning and plasmid amplification. E. coli DH5 $\alpha$ was grown at $37{ }^{\circ} \mathrm{C}$ with constant shaking in Luria-Bertani Broth supplemented with 100 $\mu \mathrm{g} \mathrm{mL}^{-1}$ of ampicillin for plasmid amplification. Y. lipolytica Po1f, a leucine and uracile auxotrophic strain devoid of any secreted protease activity, was used as the base strain for all studies [22]. The strains and plasmids used in the study are listed in Table 1.

YPD media was prepared with $20 \mathrm{~g} \mathrm{~L}^{-1}$ glucose, $10 \mathrm{~g} \mathrm{~L}^{-1}$ yeast extract and $20 \mathrm{~g}$ $\mathrm{L}^{-1}$ tryptone. Selective YNB-Ura media was prepared with $20 \mathrm{~g} \mathrm{~L}^{-1}$ glucose, $6.7 \mathrm{~g} \mathrm{~L}^{-1}$ yeast nitrogen base (without amino acids) (Difco), $0.77 \mathrm{~g} \mathrm{~L}^{-1}$ CSM-Ura (Complete Supplement Mixture minus uracil) and $20 \mathrm{~g} \mathrm{~L}^{-1}$ agar. Fermentation media was prepared with $50 \mathrm{~g} \mathrm{~L}^{-1}$ glucose, $9 \mathrm{~g} \mathrm{~L}^{-1}$ yeast extract, $8 \mathrm{~g} \mathrm{~L}^{-1}$ yeast nitrogen base (without amino acids) (Difco).

Shake flask culture was performed in $500 \mathrm{~mL}$ flask containing $100 \mathrm{~mL}$ fermentation media. Generally, yeast seed from frozen stocks were inoculated into 5 $\mathrm{mL}$ YPD media at $28{ }^{\circ} \mathrm{C}$ at $180 \mathrm{rpm}$. Overnight culture was inoculated into $50 \mathrm{~mL}$ YPD media at $28{ }^{\circ} \mathrm{C}$ at $180 \mathrm{rpm}$ in $250 \mathrm{~mL}$ shake flask for $24 \mathrm{~h}$. Yeast cells were re-inoculated into $50 \mathrm{~mL}$ YPD media with inoculation rate of 5\% under the same condition with an optical density of 0.1 and cultivated during $48 \mathrm{~h}$. The culture seed were inoculated into fermentation media with an inoculation rate of $10 \%$ for $72 \mathrm{~h}$ $\left(28{ }^{\circ} \mathrm{C}, 180 \mathrm{rpm}\right)$, after which $\mathrm{pH}$, biomass and glucose content were measured, and fatty acid profiles were analyzed, every $24 \mathrm{~h}$. 


\subsection{DNA manipulation}

To prepare individual gene expression cassettes, yeast promoters (TEF1p, 406 bp; FBA1p, $800 \mathrm{bp}$ ) and terminators (LIP2t, $563 \mathrm{bp}$; XPR2t, $422 \mathrm{bp}$ ) were de novo synthesized. The functional $\Delta-6$ desaturase, $\Delta-6$ elongase and $\Delta-5$ desaturase encoding genes from M. alpina were codon-optimized (Genscript, Nanjing, China). All the codon-optimized genes used are listed in Supplementary Table 1. The selective marker URA3 was de novo synthesized. The integration site $28 \mathrm{~S}$ rDNA coding sequence was PCR-amplified from the genomic DNA isolated from Y. lipolytica Po1f. All the primers used are listed in Supplementary Table 2. Each pathway part (over 200 ng), including promoter, functional coding gene, terminator, selective marker and integration site, was prepared by overlap extension PCR (OE-PCR) according to a previously described procedure [23]. Specifically, the first gene cassette fragment was amplified with the 28s rDNA (L)-F and LIP2t-R primer, the second gene cassette fragment was amplified with the FBAp-F and XPRt-R primer, the third gene cassette fragment was amplified with the TEFp-F and LIP2t-R primer, the fourth gene cassette fragment was amplified with the URA3-F and 28s rDNA (R)-R primer. The PCR products of each individual gene expression cassette, obtained via OE-PCR, were ligated to pMD18-T vector for cloning and sequencing. A DNA mixture of individual cassettes (over $300 \mathrm{ng}$ ) was used to transform Y. lipolytica Po1f strain cells.

\subsection{Yeast transformation}

Transformation of $Y$. lipolytica was performed by the modified lithium acetate procedure previously described [24-25]. Briefly, Y. lipolytica Polf was inoculated 
from glycerol stock directly into $10 \mathrm{~mL}$ YPD media and shaken at $28{ }^{\circ} \mathrm{C}$ at $180 \mathrm{rpm}$ for $24 \mathrm{~h}$. Yeast cells were inoculated with a ratio of $5 \%$ into $50 \mathrm{~mL}$ YPD media and shaken at $28{ }^{\circ} \mathrm{C}$ at $180 \mathrm{rpm}$ until $\mathrm{OD}_{600}$ reached 0.7 . Then, a sample of $2.5 \mathrm{~mL}$ ice-precooled $1 \mathrm{M}$ hydroxyurea was added into the culture. Yeast cells were shaken and harvested when $\mathrm{OD}_{600}$ reached 1.0 to 1.5 , by centrifugation at $4000 \mathrm{rpm}$ for 10 min. Yeast cells were washed twice in ice-precooled sterile water. Then, yeast cells were resuspended in $50 \mathrm{~mL}$ ice-precooled $100 \mathrm{mM} \mathrm{LiOAc}(\mathrm{pH} \mathrm{6.0)}$ and incubated at $28{ }^{\circ} \mathrm{C}$ for $60 \mathrm{~min}$ without shaking. Next, yeast cells were resuspended and rinsed in different conditions (the first time with $25 \mathrm{~mL}$ of ice-precooled $100 \mathrm{mM} \mathrm{LiOAc}(\mathrm{pH}$ 6.0) by centrifugation at $4000 \mathrm{rpm}$ for $10 \mathrm{~min}$, the second time with $2 \mathrm{~mL}$ of ice-precooled $100 \mathrm{mM} \mathrm{LiOAc}(\mathrm{pH}$ 6.0) by centrifugation at $4000 \mathrm{rpm}$ for $10 \mathrm{~min}$, the third time with $1 \mathrm{~mL}$ of ice-precooled $100 \mathrm{mM} \mathrm{LiOAc}(\mathrm{pH} \mathrm{6.0)}$ by centrifugation at 4 $000 \mathrm{rpm}$ for $10 \mathrm{~min}$ ). At last, yeast cells were resuspended in $100 \mu \mathrm{L}$ of $100 \mathrm{mM}$ LiOAc ( $\mathrm{pH}$ 6.0) and used for transformation directly. A sample of 2-5 $\mu$ g of DNA mixture of each gene expression cassette was added to transformation cells, followed by $10 \mu \mathrm{L}$ of $100 \mathrm{mg} \mathrm{mL}^{-1}$ boiled salmon sperm DNA. Yeast cells were incubated at $28{ }^{\circ} \mathrm{C}$ for $15 \mathrm{~min}$ with shaking at $150 \mathrm{rpm}$, followed by addition of $720 \mu \mathrm{L}$ of PEG buffer (40\% PEG-3500, $100 \mathrm{mM}$ LiOAc, pH 6.0) and $100 \mu \mathrm{L}$ of $1 \mathrm{M}$ dithiothreitol. Yeast cells were incubated at $28{ }^{\circ} \mathrm{C}$ for 60 min with shaking at $220 \mathrm{rpm}$, then heat shocked for $10 \mathrm{~min}$ at $39{ }^{\circ} \mathrm{C}$, spun down and resuspended in $1 \mathrm{~mL}$ of pre-cooling 100 mM LiOAc (pH 6.0). $200 \mu \mathrm{L}$ of cells were grown on YNB-Ura plates at $28{ }^{\circ} \mathrm{C}$ for $2-5$ days for selection of the engineered transformants. 


\subsection{PCR analysis of the assembled pathway}

Genomic DNAs were extracted from the original $Y$. lipolytica Po1f and the engineered $Y$. lipolytica YL 6-1 strains, following shake flask cultivation for $48 \mathrm{~h}$, using a yeast genomic DNA kit (Omega Bio-tech, USA). For ARA biosynthesis from $\Delta-6$ desaturation pathway, confirmation of the correct DNA assembly was performed by PCR amplification of each structural gene from the genomic DNAs. All the primers used are listed in Supplementary Table 2.

\subsection{Copy number analysis}

To determine the relative copy number of the gene expression cassettes of pathway assembly in the engineered $Y$. lipolytica, qPCR analysis was performed on the genomic DNA using ACT1 as the internal control. Genomic DNAs were extracted from the original $Y$. lipolytica Po1f and the engineered $Y$. lipolytica YL 6-1 strains, following cultivation for $48 \mathrm{~h}$, using a yeast genomic DNA kit (Omega Bio-tech, USA). qPCR was performed on an ABI 7900 instrument (Applied Biosystems, Foster City, CA, USA) and the primers used are listed in Supplementary Table 1. Fold changes were determined by the $2^{-\Delta \Delta \mathrm{Ct}}$ method and normalized to the ACT1 gene. All experiments were performed in triplicate.

\subsection{Analysis of fatty acid profiles}

Fatty acid methyl esters of $Y$. lipolytica strains were prepared as previously described [26]. GC analysis of fatty acid methyl esters was performed with GC-FID (GC-2010, Shimadzu, Japan) equipped with a capillary column (DB-23, $60 \mathrm{~m} \times 0.22$ $\mathrm{mm}$ ) to analyze fatty acid profiles of sample. Using nitrogen as the carrier gas, a 
sample of $1 \mu \mathrm{L}$ was injected with the injector at $250^{\circ} \mathrm{C}$. The column temperature was raised from 100 to $196{ }^{\circ} \mathrm{C}$ at $25{ }^{\circ} \mathrm{C} \min ^{-1}$ and then increased to $220{ }^{\circ} \mathrm{C}$ at $2{ }^{\circ} \mathrm{C} \min ^{-1}$, keeping this temperature for $6 \mathrm{~min}$. The FID detector temperature was set to $280{ }^{\circ} \mathrm{C}$. Fatty-acid standards for methyl palmitate (C16:0), methyl stearate (C18:0), methyl oleate (C18:1), methyl linoleate (C18:2), methyl octadecatrienoate (C18:3), methyl eicosatrienoate (C20:3) and methyl arachidonate (C20:4) were purchased from Sigma Aldrich and used as standard to identify fatty-acid peaks. Total lipid content of samples with different fatty-acid methylesters was calculated using fatty-acid standards.

\section{$2.7 \mathrm{pH}$, biomass and residual glucose quantification}

pH was measured using a $\mathrm{pH}$ electrode (Mettler-Toledo, Urdorf, Switzerland). Cell biomasses were harvested by centrifugation at $12000 \mathrm{rpm}$ for $10 \mathrm{~min}$, washed twice with distilled water and dried at $70{ }^{\circ} \mathrm{C}$ to a constant weight. Glucose content was measured by a biosensor with glucose oxidase electrode (SBA-40C, Institute of Biology, Shandong Academy of Sciences, China).

\section{Results and discussion}

3.1 Design of One-step pathway assembly for arachidonic acid production in

\section{Yarrowia lipolytica}

We provide here a proof of concept of the possibility and efficiency of in vivo one-step pathway assembly in a non-conventional yeast chromosome, using $Y$. lipolytica as chassis. Generally, with rDNA as integration locus, a metabolic pathway assembly experiment is designed as shown in Fig. 1. Briefly, each functional gene 
cassette, consisting of a promoter, a structural gene and a terminator, is synthesized via an OE-PCR step. The 5'-end of the first gene cassette is designed to present homology with the chromosome loci of rDNA in order to promote integration, while the 3'-end of the first gene cassette is designed to present homology with the 5'-end of the second cassette, and so-on, and the 3'-end of the last gene cassette is designed to present homology with the chromosome loci of rDNA. Each gene cassette is designed to share a same sequence overlap with the neighboring one, in order to promote their ordered integration by homologous recombination into the chromosome.

We use here ARA biosynthesis as a case study for this one-step pathway assembly method. ARA is synthesized in engineered $Y$. lipolytica via a three steps reaction: the first step is the $\Delta-6$ desaturation of linoleic acid to gamma-linolenic acid, the second step is the $\Delta-6$ elongation of gamma-linolenic acid to dihomo-gamma-linolenic acid and the last step is the $\Delta-5$ desaturation of dihomo-gamma-linolenic acid to ARA (Fig. 2). With Y. lipolytica as a chassis, targeted ARA has previously been synthesized by the DuPont Company using integrative plasmids constructed via the classic restriction digestion and ligation method [27]. As shown in Supplementary Fig. 1, our scheme of in vivo one-step assembly is capable of reducing the time and cost of constructing the entire biosynthesis pathway, compared with the classic assembly strategy. In our study, the ARA biosynthesis pathway, designed via in vivo one-step assembly, consists of four gene cassettes, namely $28 \mathrm{~S}$ rDNA (L)-TEF1p- $\Delta-6$ desaturase-LIP2t, FBAp- $\Delta-6$ 
elongase-XPR2t, TEF1p- $\Delta-5$ desaturase-LIP2t, URA3-28S rDNA(R). The three ARA synthesis pathway genes, $\Delta-6$ desaturase, $\Delta-6$ elongase and $\Delta-5$ desaturase encoding genes from M. alpina, were codon-optimized for expression in $Y$. lipolytica. Moreover, a Kozak sequence was added before each structural gene to improve the transcription level.

In addition, rDNA sequence was selected as integration target sequence for metabolic pathway integration. We choose in our study to use rDNA as a target for integration, preferably to "zeta" sequences that are often used for $Y$. lipolytica engineering, since Po1f strain is devoid of Ylt1 retrotransposon and of dispersed repeated "zeta" sequences [28]. Although the DNA fragments with "zeta" borders can be integrated at random in Y. lipolytica strain (no "zeta") via a non-homologous end joining process, the transformation frequency was low [29-31]. Interestingly, the partial functional cassettes of mixtures were detected in the transformants based on PCR analysis (data not shown). That is maybe that the partial genes were integrated in non-homologous locations. Meanwhile, it was noticed that the transformation efficiency changed as a function of gene concentration in the transformation mix. Especially, if the gene concentration was exceeded over $5 \mathrm{ng}$, the transformation efficiency declined (data not shown). The integration events in our research were similar to previously report [31]. On the basis of the PCR screening, a positive engineered $Y$. lipolytica strain, YL 6-1, was selected for measurement of ARA production. As shown in Supplementary Fig. 2, the engineered Y. lipolytica YL 6-1 strain was able to produce a significant ARA amount, during a $48 \mathrm{~h}$ shake flask 
fermentation, whereas the original $Y$. lipolytica Polf strain was not able to synthesize ARA.

\subsection{Relation between the length of overlapping region and assembly efficiency}

To test the effect of various lengths of overlapping regions on the efficiency of pathway assembly, different combinational lengths of overlaps were designed. As shown in Table 2, for the type one assembly design, overlaps between each gene cassette were 50-60 bp and homology with chromosomal rDNA sequence was $300 \mathrm{bp}$. The total size of the three genes together with the URA3 marker was nearly $9.2 \mathrm{~kb}$. For the type two, overlaps between cassettes were 50-60 bp and homologous rDNA was $900-1000 \mathrm{bp}$. The total size of the three genes plus $U R A 3$ was nearly $10.4 \mathrm{~kb}$. For the type three, overlaps between cassettes were 400-500 bp and homologous rDNA was 900-1000 bp. The total size of the three genes plus URA3 was nearly $10.4 \mathrm{~kb}$. Typically, in transformation experiments performed with these DNA mixtures, 20-50 colonies appeared after $2-4$ days at $28{ }^{\circ} \mathrm{C}$. In order to confirm the correct assembly and integration of the pathway, 30 clones of each assembly type were randomly picked for genomic DNA extraction. PCR analysis was used to confirm the presence of each gene of the biosynthesis pathway, namely $\Delta-6$ desaturease, $\Delta-6$ elongase and $\Delta-5$ desaturase encoding genes (data not shown). Obviously, the highest correct assembly efficiency, of $23 \%$, was obtained with the type three assembly design. Meanwhile, the correct assembly efficiency for type one and type two reached nearly $7 \%$ and $13 \%$, respectively. It is supposed that the efficiency of pathway assembly is mainly affected by the length of overlaps. Recently, the same research on the effect of 
homology length on the frequency of homologous integration in $Y$. lipolytica was reported. Verbeke et al. [30] performed that, with homology length of $500 \mathrm{bp}$, only $2 \%$ of the frequency of homologous integration is reported in $Y$. lipolytica Pold, However, with flanking shorter regions (50,100 and $250 \mathrm{bp}$, respectively), the homologous recombination is impossible in Y. lipolytica Pold.

When we compare our results with the higher efficiency of in vivo pathway assembly (60-80\%) in S. cerevisiae, it appears that the reason of this large difference in assembly efficiency between $S$. cerevisiae and $Y$. lipolytica is that $Y$. lipolytica is a non-conventional yeast with a higher efficiency of non-homologous recombination than of homologous recombination [32]. Recently, Gao et al. [21] performed that beta-carotene is synthesized via one-step pathway assembly in $Y$. lipolytica with an assembly efficiency of $12-21 \%$. The difference in integration efficiency is maybe attributed to the different lengths of homologous sequences, the special traits of $Y$. lipolytica strains and the different biosynthesis pathway of drop-in products. To improve the efficiency of homologous recombination in Y. lipolytica, the use of strains deleted for $K U 70$ and $K U 80$ has been reported [33-34]. However, the efficiency of homologous recombination was mainly affected by the homlolgous intregration region in the engineered $Y$. lipolytica. Especially, a dramatic increase in the frequency of homologous recombination (60.0-63.3\%) was found for homologous integration sequences with a length of 750 and $1000 \mathrm{bp}$ in Y. lipolytica H222-SW2 ( $\Delta k u 70)$ [34]. Recently, the treatment of competent cells of $Y$. lipolytica with hydroxyurea, with has a function of cell cycle arrest, has been reported to increase gene targeting [25]. In the 
present study, hydroxyurea-treated yeast cells have been used to improve the efficiency of pathway assembly.

\subsection{Copy number estimation}

To verify the copy number of the heterologous genes within the entire pathway integrated into the chromosome of YL 6-1, qPCR was performed on genomic DNAs of the engineered strain. The relative copy number of $\Delta-6$ desaturase, $\Delta-6$ elongase and $\Delta-5$ desaturase encoding genes of the engineered YL 6-1 strain have been determined to be roughly 1 (Supplementary Fig. 3). In fact, with the relative copy number of $\Delta-6$ desaturase, $\Delta-6$ elongase and $\Delta-5$ desaturase encoding genes of the engineered YL 6-1 as control, the relative copy number of functional genes in Polf was determined to be roughly 0 . It is maybe that the entire ARA biosynthesis pathway is not present in Polf. A series of genetic tools, including integrative plasmids, have been developed for using $Y$. lipolytica as a chassis for various applications [11]. Generally, the integration of a heterologous gene cassette can be targeted, by using bordering regions of homology, to a chosen chromosomal site. Several loci have been used for that purpose in $Y$. lipolytica, including a single-copy gene such as $X P R 2$, the copies of tandemly repeated sequences of rDNA or the dispersed copies of repeated elements such as the "zeta" sequence of Ylt1 retrotransposon [35]. Notably, there are at least 100 potential "zeta" sequence integration sites and more than 200 copies of rDNA clusters in $Y$. lipolytica [35-36].

Interestingly, there is a relation between the copy number and the selection marker convention rate. Previously, the integrants carrying up to 60 copies of 
transformants were obtained via the defective URA3 gene in rDNA-based Y. lipolytica strain [37]. Especially, when using the ura3d4 allele for selection, the higher multicopy transformants were obtained, regardless of whether the "zeta" or rDNA, or the single copy XPR2 locus, were used as targeting sites [29]. As demonstrated, the multiple integrations are generally obtained only when a defective selection marker is used, regardless of the number of copy of the target used [30-31]. Therefore, obtaining only one integrated copy of the new pathway was the result expected in our case, since the fully functional URA3 gene has been used as selective marker. Previously, another pathway engineering experiment using a non-defective marker and homologies of $300 \mathrm{bp}$ with $28 \mathrm{~S}$ rDNA has also lead to single copy integration $[38]$.

\subsection{Stability assessment}

In the following step, genomic stability of the engineered $Y$. lipolytica strain YL 6-1 capable of producing ARA was assessed under non-selection culture. The engineered YL 6-1 strain was cultivated over 40 generations. Based on PCR analysis, shown in Supplementary Fig. 4, the fortieth generation of the engineered Y. lipolytica YL 6-1 was found to have conserved the three heterologous gene of the engineered pathway. Accordingly, it had also been showed previously that engineered Y. lipolytica with $28 \mathrm{~S}$ rDNA as target site for pathway integration was stable even over 40 generations [38]. In the present study, we demonstrated that our method allowed high assembly efficiency and long-term genetic stability of the engineered $Y$. lipolytica strain. 


\subsection{Fermentation performance of engineered Yarrowia lipolytica}

With linoleic acid (C18:2) as a substrate, the ARA can be accumulated in $Y$. lipolytica via the in vivo one-step assembled ARA biosynthesis pathway. To verify the possible effect of the de novo ARA biosynthesis pathway on the fatty acid profiles of $Y$. lipolytica, GC analysis of the main fatty acid profiles, including palmitic acid (C16:0), stearic acid (C18:0), oleic acid (C18:1), linoleic acid (C18:2), gamma-linolenic acid (C18:3), dihomo-gamma-linolenic acid (C20:3) and arachidonic acid (C20:4), was performed.

As shown in Fig. 3, the C16:0 and the C18:0 content of the original Po1f strain was slightly higher than those of the engineered YL 6-1 under fermentation at $72 \mathrm{~h}$, respectively. Interestingly, when compared with the original Polf strain, the engineered YL 6-1 strain exhibited a higher consumption rate of C18:1. The C18:2 content of the original and the engineered strain was detected, respectively. Especially, when compared with that of the original Po1f strain, the C18:2 content of the engineered YL 6-1 strain was significantly lower. Due to the absence of native $\Delta-6$ desaturase, $\Delta-6$ elongase and $\Delta-5$ desaturase functions, $Y$. lipolytica is not able to synthesize C20:4 and intermediates C18:3 and C20:3. As shown in Fig. 3, Po1f was not able to produce $\mathrm{C} 18: 3$. Whereas, using $\mathrm{C} 18: 2$ as a substrate, $\mathrm{C} 18: 3$ was synthesized in the engineered $Y$. lipolytica strain via the novel ARA biosynthesis pathway. For YL 6-1 strain, the C18:3 content reached to $2.8 \%$ of total fatty acids at

72 h. Recently, we have constructed an engineered $Y$. lipolytica capable of producing C18:3 (6\% of TFA) under the optimized fermentation process [39]. Using C18:3 as a 
substrate, this engineered strain was thus able to produce C20:3 via the novel ARA biosynthesis pathway. Especially, the C20:3 content of the engineered YL 6-1 strain was at $0.7 \%$ of total fatty acids at $72 \mathrm{~h}$. In contrast to the original Polf strain, the engineered YL 6-1 strain was able to synthesize C20:4. Especially, a high C20:4 content of $0.4 \%$ of total fatty acids was produced at $72 \mathrm{~h}$. With the novel functions of the heterologous ARA biosynthesis pathway, the targeted ARA and intermediates, gamma-linolenic and dihomo-gamma-linolenic acid, were produced in the engineered Y. lipolytica. It was concluded that the ARA biosynthesis pathway from M. alpina can function efficiently in Y. lipolytica.

With glucose at $50 \mathrm{~g} \mathrm{~L}^{-1}$ as an initial carbon substrate, the $\mathrm{pH}$ value is shown in Fig. 4. Obviously, the $\mathrm{pH}$ value of the fermentation culture in the engineered YL 6-1 strain was higher than that of the original Polf strain at 72 h. Especially, the $\mathrm{pH}$ value of the fermentation culture in the original Polf was 2.1 at $72 \mathrm{~h}$. For the fermentation culture in the engineered YL 6-1, the $\mathrm{pH}$ value was 3.3 at $72 \mathrm{~h}$. In fact, there is a relation between the $\mathrm{pH}$ and the secreted organic acids in the fermentation culture. Especially, citric acid and malic acid are viewed as important metabolic intermediates with potential for providing the precursors of fatty acid biosynthesis. Future work is to further evaluate the metabolic flux of the engineered and orignal strians, based on the analysis of organic acids production in the fermentation culture. Moreover, the biomass titer of the engineered YL 6-1 strain was significantly higher than that of the original Po1f strain (10.2 vs $6.9 \mathrm{~g} \mathrm{~L}^{-1}$ ) (Fig. 4). As shown in Fig. 4, the rate of glucose consumption of the engineered YL 6-1 strain was thus slightly higher than that of the 
original Polf strain. Recently, it was reported that the leucine can be viewed as an intracellular trigger to promote lipogenesis [40]. Although the leucine auxotrophic strains were used in this research, compared to the original Polf, the engineered strain YL 6-1 performed a higher glucose uptake and biomass production. It was concluded that more cellular substrates maybe are diverted toward the biomass production and lipogenesis in the leucine auxotrophic strain YL 6-1. Especially, the heterologous ARA biosynthesis pathway was able to direct the metabolic flux to the desired product. Based on our analysis of the fermentation performances of the original and engineered strain, it was demonstrated that the ARA biosynthesis pathway in $M$. alpina can function well in Y. lipolytica without adverse effects.

\section{Conclusions}

In this work, with ARA biosynthesis as a case study, the in vivo one-step pathway assembly strategy was successfully developed for the non-conventional yeast $Y$. lipolytica. Based on the analysis of the effect of different overlapping regions, this study showed that the efficiency of pathway assembly was mainly affected by the length of overlaps. Especially, the highest efficiency (nearly 23\%) was obtained when using overlaps of roughly $1 \mathrm{~kb}$, in accordance to previous data on homologous recombination in $Y$. lipolytica [41]. Furthermore, the accumulation of ARA and intermediate gamma-linolenic and dihomo-gamma-linolenic acid between the original and engineered $Y$. lipolytica was demonstrated. Our results show that the engineered strain can produce ARA, up to a content of $0.4 \%$ of total fatty acids, during the fermentation. In addition, this study showed that the newly integrated heterologous 
ARA biosynthesis pathway was stably maintained through at least 40 generations and that it was sufficient to allow obtaining a higher titer of biomass during fermentation. One can hypothesize that this increased growth performance of the ARA pathway bearing cells could contribute, as a selective advantage, to the maintenance of this modification through the generations, during long term cultivation. Altogether, this study showed that the one-step in vivo assembly and integration method enables reliable assembly of many DNA parts in Y. lipolytica via a rapid and convenient workflow. Future work focusing on the utilization of other integration sites, such as MFE locus and Pex10 locus, should further induce and improve the accumulation of ARA. With the development of synthetic biology, the CRISPR-Cas9 genome editing tools for Y. lipolytica have recently been expanded [42-43]. Combined with these well-developed genetic tools, the one-step in vivo assembly and integration method will facilitate the use of non-conventional yeasts in many applications. 


\section{References}

[1] J. Nielsen, J.D. Keasling, Engineering Cellular Metabolism, Cell. 164 (2016) $1185-1197$.

[2] P.P. Peralta-Yahya, F. Zhang, S.B. del Cardayre, J.D. Keasling, Microbial engineering for the production of advanced biofuels, Nature. 488 (2012) $320-328$.

[3] R. Jain, X. Sun, Q. Yuan, Y. Yan, Systematically engineering Escherichia coli for enhanced production of 1,2-propanediol and 1-propanol, ACS. Synth. Biol. 4 (2015) 746-756.

[4] K. Thodey, S. Galanie, C.D Smolke, A microbial biomanufacturing platform for natural and semisynthetic opioids, Nat. Chem. Biol. 10 (2014) 837-844.

[5] N. Bati, E.G. Hammond, B.A. Glatz, Biomodification of fats and oils: trials with Candida lipolytica, J. Am. Oil. Chem. Soc. 61 (1984) 1743-1746.

[6] R. Ledesma-Amaro, J.M. Nicaud, Metabolic engineering for expanding the engineering for expanding the substrate range of Yarrowia lipolytica, Trends. Biotechnol. 2016, doi: 10.1016/j.tibtech.2016.04.010.

[7] H.H. Liu, X.J. Ji, H. Huang, Biotechnological applications of Yarrowia lipolytica: past, present and future, Biotechnol. Adv. 33 (2015) 1522-1546.

[8] C. Madzak, Yarrowia lipolytica: recent achievements in heterologous protein expression and pathway engineering, Appl. Microbiol. Biotechnol. 99 (2015) $4559-4577$.

[9] H. Guo, P. Liu, C. Madzak, G. Du, J. Zhou, J. Chen, Identification and application 
of keto acids transporters in Yarrowia lipolytica, Sci. Rep. 5 (2015) 8138.

[10] P. Ferreira, M. Lopes, M. Mota, I. Belo, Oxygen mass transfer impact on citric acid production by Yarrowia lipolytica from crude glycerol, Biochem. Eng. J. 110 (2016) 35-42.

[11] F. Matthäus, M. Ketelhot, M. Gatter, G. Barth, Production of lycopene in the non-carotenoid-producing yeast Yarrowia lipolytica, Appl. Environ. Microbiol. 80 (2014) 1660-1669.

[12] Z. Xue, P.L. Sharpe, S.P. Hong, N.S. Yadav, D. Xie, D.R. Short, H.G. Damude, R.A. Rupert, J.E. Seip, J. Wang, D.W. Pollak, M.W. Bostick, M.D. Bosak, D.J. Macool, D.H. Hollerbach, H. Zhang, D.M. Arcilla, S.A. Bledsoe, K. Croker, E.F. McCord, B.D. Tyreus, E.N. Jackson, Q. Zhu, Production of omega-3 eicosapentaenoic acid by metabolic engineering of Yarrowia lipolytica, Nat. Biotechnol. 31 (2013):734-740.

[13] G. Wang, X. Xiong, R. Ghogare, P. Wang, Y. Meng, S. Chen, Exploring fatty alcohol-producing capability of Yarrowia lipolytica, Biotechnol. Biofuels. 9 (2016) 107.

[14] P. Xu, K. Qiao, W.S. Ahn, G. Stephanopoulos, Engineering Yarrowia lipolytica as a platform for synthesis of drop-in transportation fuels and oleochemicals, Proc. Natl. Acad. Sci. U. S. A. 113 (2016) 10848-10853.

[15] X.J. Ji, L.J. Ren, Z.K. Nie, H. Huang, P.K. Ouyang, Fungal arachidonic acid-rich oil: research, development and industrialization, Crit. Rev. Biotechnol. 34 (2014) 197-214. 
[16] J.R. Petrie, P. Shrestha, S. Belide, M.P. Mansour, Q. Liu, Horne J, P.D. Nichols, S.P. Singh, Transgenic production of arachidonic acid in oilseeds, Transgenic. Res. 21 (2012):139-147.

[17] F. Beaudoin, L.V. Michaelson, S.J. Hey, M.J. Lewis, P.R. Shewry, O. Sayanova, J.A. Napier, Heterologous reconstitution in yeast of the polyunsaturated fatty acid biosynthetic pathway, Proc. Natl. Acad. Sci. U. S. A. 97 (2000) 6421-6426.

[18] R.E. Cobb, J.C. Ning, H. Zhao, DNA assembly techniques for next-generation combinatorial biosynthesis of natural products, J. Ind. Microbiol. Biotechnol. 41 (2014) 469-477.

[19] P. Xu, A. Vansiri, N. Bhan, M.A. Koffas, ePathBrick: a synthetic biology platform for engineering metabolic pathways in E. coli, ACS Synth. Biol. 1 (2012) 256-266.

[20] Z. Shao, H. Zhao, H. Zhao, DNA assembler, an in vivo genetic method for rapid construction of biochemical pathways, Nucleic Acids Res. 37 (2009) e16.

[21] S. Gao, L. Han, L. Zhu, M. Ge, S. Yang, Y. Jiang, D. Chen, One-step integration of multiple genes into the oleaginous yeast Yarrowia lipolytica, Biotechnol. Lett. 36 (2014) 2523-2528.

[22] C. Madzak, B. Tréton, S. Blanchin-Roland, Strong hybrid promoters and integrative expression/secretion vectors for quasi-constitutive expression of heterologous proteins in the yeast Yarrowia lipolytica, J. Mol. Microbiol. Biotechnol. 2 (2000) 207-216.

[23]. R.M. Horton, H.D. Hunt, S.N. Ho, J.K. Pullen, L.R. Pease, Engineering hybrid 
genes without the use of restriction enzymes-gene-splicing by overlap extension, Gene. 77 (1989) 61-68.

[24] D.C. Chen, J.M. Beckerich, C. Gaillardin, One-step transformation of the dimorphic yeast Yarrowia lipolytica, Appl. Microbiol. Biotechnol. 48 (1997) $232-235$.

[25] V. Tsakraklides, E. Brevnova, G. Stephanopoulos, A.J. Shaw, Improved gene targeting through cell cycle synchronization, PLoS One. 10 (2015) e0133434.

[26] E.B. Cahoon, K.G. Ripp, S.E. Hall, A.J. Kinney, Formation of conjugated delta8, delta10-double bonds by delta12-oleic-acid desaturase-related enzymes: biosynthetic origin of calendic acid, J. Biol. Chem. 276 (2001) 2637-2643.

[27] H.G. Damude, P.J. Gillies, D.J. Macool, W. Pollak, J.J. Ragghianti, Z.X. Xue, N.S. Yadav, H.X. Zhang, Q.N. Zhu, High arachidonic acid producing strains of Yarrowia lipolytica, United. States. Patent. 2009, US 7, 588, 931.

[28] L. Liu, H.S. Alper, Draft genome sequence of the oleaginous yeast Yarrowia lipolytica PO1f, a commonly used metabolic engineering host, Genome Announc. 2 (2014) e00652-14.

[29] T. Juretzek, M. Le Dall, S. Mauersberger, C. Gaillardin, G. Barth, J.M. Nicaud, Vectors for gene expression and amplification in the yeast Yarrowia lipolytica, Yeast. 18 (2001) 97-113

[30] G. Pignède, H.J. Wang, F. Fudalej, M. Seman, C. Gaillardin, J.M. Nicaud, Autocloning and amplification of LIP2 in Yarrowia lipolytica. Appl. Environ. Microbiol. 66 (2000) 3283-3289. 
[31] F. Bordes, F. Fudalej, V. Dossat, J.M. Nicaud, A. Marty, A new recombinant protein expression system for high-throughput screening in the yeast Yarrowia lipolytica, J. Microbiol. Methods. 70 (2007) 493-502.

[32] G.F. Richard, A. Kerrest, I. Lafontaine, B. Dujon, Comparative genomics of hemiascomycete yeasts: genes involved in DNA replication, repair, and recombination, Mol. Biol. Evol. 22 (2005) 1011-1023.

[33] Verbeke J, A. Beopoulos, J.M. Nicaud, Efficient homologous recombination withshort length flanking fragments in Ku70 deficient Yarrowia lipolytica strains, Biotechnol. Lett. 35 (2013) 571-576.

[34] A. Kretzschmar, C. Otto, M. Holz, S. Werner, L. Hübner, G. Barth, Increased homologous integration frequency in Yarrowia lipolytica strains defective in non-homologous end-joining, Curr. Genet. 59 (2013) 63-72.

[35] N. Schmid-Berger, B. Schmid, G. Barth. Ylt1, a highly repetitive retrotransposon in the genome of the dimorphic fungus Yarrowia lipolytica, J. Bacteriol. 176 (1994) 2477-2482.

[36] S. Casarégola, C. Feynerol, M. Diez, P. Fournier, C. Gaillardin, Genomic organization of the yeast Yarrowia lipolytica, Chromosoma. 106 (1997) 380-390.

[37] M.T. Le Dall, J.M. Nicaud, C. Gaillardin, Multiple-copy integration in the yeast Yarrowia lipolytica, Curr. Genet. 26 (1994) 38-44.

[38] E. Celińska, W. Grajek, A novel multigene expression construct for modification of glycerol metabolism in Yarrowia lipolytica, Microb. Cell. Fact. 12 (2013) 102.

[39] M.L. Sun, C. Madzak, H.H. Liu, P. Song, L.J. Ren, H. Huang, X.J. Ji, 
Engineering Yarrowia lipolytica for efficient $\gamma$-linolenic acid production, Biochem. Eng. J. 117 (2017) 172-180.

[40] J. Blazeck, A. Hill, L. Liu, R. Knight, J. Miller, A. Pan, P. Otoupal, H.S. Alper, Harnessing Yarrowia lipolytica lipogenesis to create a platform for lipid and biofuel production, Nat. Commun. 5 (2014) 3131.

[41] G. Barth, C. Gaillardin, Yarrowia lipolytica, in: Wolf, K. (Eds.), Nonconventional Yeasts in Biotechnology, Springer-Verlag, Berlin Heidelberg, 1996, pp. 313-388.

[42] C.M. Schwartz, M.S. Hussain, M. Blenner, I. Wheeldon, Synthetic RNA polymerase III promoters facilitate high-efficiency CRISPR-Cas9-mediated genome editing in Yarrowia lipolytica, ACS. Synth. Biol. 5 (2016) 356-359.

[43] S. Gao, Y. Tong, Z. Wen, L. Zhu, M. Ge, D. Chen, Y. Jiang, S. Yang, Multiplex gene editing of the Yarrowia lipolytica genome using the CRISPR-Cas9 system, J. Ind. Microbiol. Biotechnol. 43 (2016) 1085-1093. 


\section{Tables and Figure Legends}

Table 1: Plasmids and strains used in this study.

Table 2. The efficiency of in vivo one-step assembly and integration of arachidonic acid biosynthesis pathway with different lengths of overlap in Yarrowia lipolytica. (For each type, 30 colonies were randomly picked and analyzed.)

Fig. 1. A one-step method for assembly and intregration of arachidonic acid biosynthesis pathway using in vivo homologous recombination in Yarrowia lipolytica. OE-PCR, overlap extension PCR.

Fig. 2. Arachidonic acid biosynthesis via three steps reaction.

Fig. 3. Fatty acid profiles of the original strain Yarrowia lipolytica Po1f and the recombinant strain Y. lipolytica YL 6-1. C16:0, palmitic acid; C18:0, stearic acid; C18:1, oleic acid; C18:2, linoleic acid; C18:3, gamma-linolenic acid; C20:3, dihomo-gamma-linolenic; C20:4, arachidonic acid.

Fig. 4. Fermentation performance of the original strain Yarrowia lipolytica Polf and the recombinant strain $Y$. lipolytica YL 6-1. 
Table 1.

\begin{tabular}{|c|c|c|}
\hline Strains & Genotype and/or heterologous expression cassette & Reference \\
\hline \multicolumn{3}{|l|}{ E. coli } \\
\hline \multirow[t]{3}{*}{$\mathrm{DH} 5 \alpha$} & fhuA2 (argF-lacZ) U169 phoA glnV44 p80 A(lacZ)M15 & \\
\hline & & Vazyme Biotech \\
\hline & gyrA96 recAl relAl endAl thi- 1 hsdR17 & \\
\hline \multicolumn{3}{|l|}{ Y. lipolytica } \\
\hline Polf & MatA, leu2-270, ura3-302, xpr2-322, axp1-2 & {$[20]$} \\
\hline \multirow[t]{3}{*}{ YL 6-1 } & MatA, leu2-270, ura3-302, xpr2-322, axp1-2, URA3 & \\
\hline & & This work \\
\hline & $T E F \mathrm{p}-\triangle-6-\mathrm{D}-L I P 2 \mathrm{t}, F B A \mathrm{p}-\Delta-6-\mathrm{E}-X P R 2 \mathrm{t}, T E F \mathrm{p}-\Delta-5-\mathrm{D}-L I P 2 \mathrm{t}$ & \\
\hline \multicolumn{3}{|l|}{ Plasmid } \\
\hline pMD18-T & & TAKARA \\
\hline pMD18-YL-1 & rDNA (L)-TEFp- $\triangle-6-\mathrm{D}-L I P 2 \mathrm{t} 2$ & This work \\
\hline pMD18-YL-2 & $F B A \mathrm{p}-\triangle-6-\mathrm{E}-X P R 2 \mathrm{t}$ & This work \\
\hline pMD18-YL-3 & $T E F \mathrm{p}-\triangle-5-\mathrm{D}-L I P 2 \mathrm{t}$ & This work \\
\hline pMD18-YL-4 & URA3- rDNA (R) & This work \\
\hline
\end{tabular}

Codon-optimized M. alpina genes: $\Delta-6-\mathrm{D}, \Delta-6$ desaturase; $\Delta-6-\mathrm{E}, \Delta-6$ elongase; $\Delta-5-\mathrm{D}, \Delta-5$

desaturase. 
Table 2.

\begin{tabular}{cccc}
\hline $\begin{array}{c}\text { Length of various } \\
\text { overlap }\end{array}$ & Total colonies & Correct colonies & \\
(PCR analysis) & (PCR analysis) & Cumulative probability (\%) \\
Type one & 30 & 2 & 6.7 \\
Type two & 30 & 4 & 13.3 \\
Type three & 30 & 7 & 23.3 \\
\hline
\end{tabular}




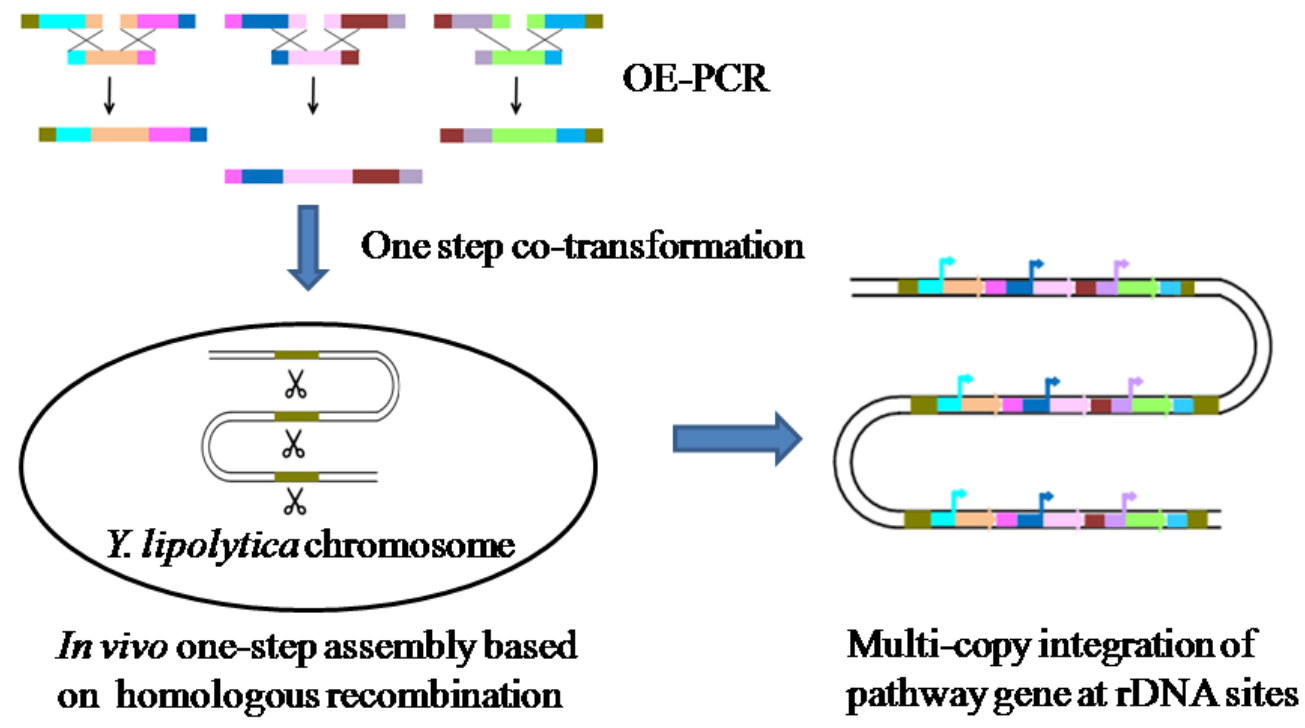

Fig. 1 


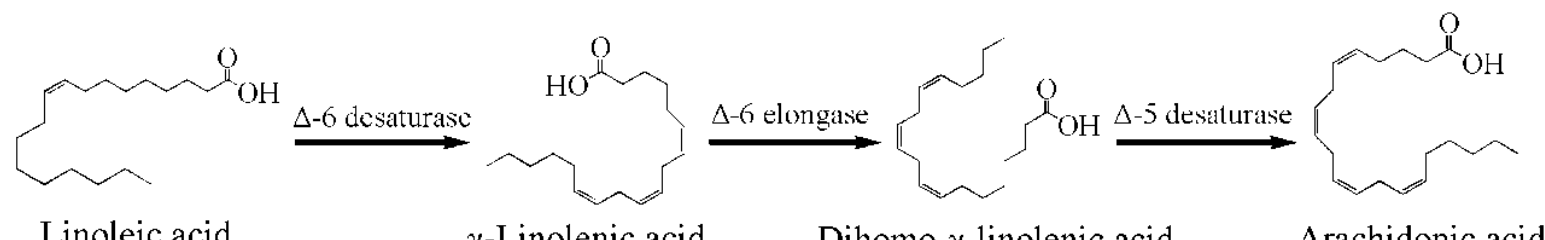

Fig. 2 


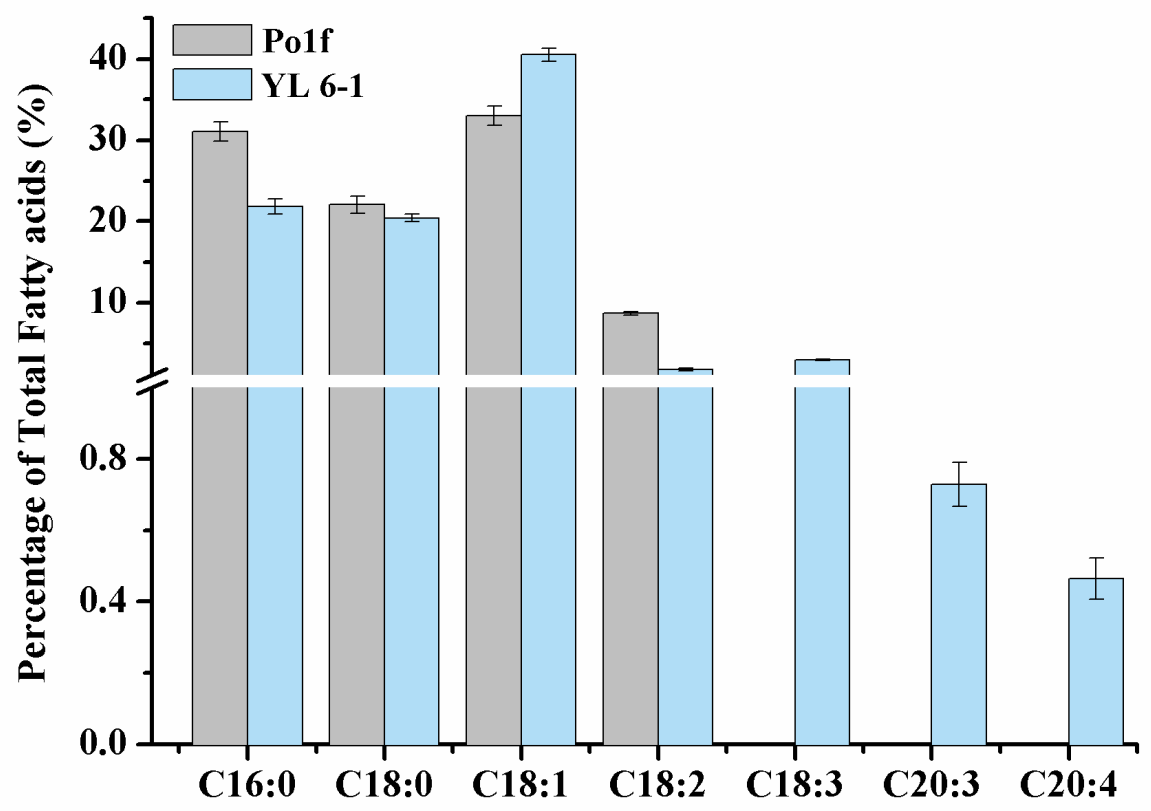

Fig. 3 


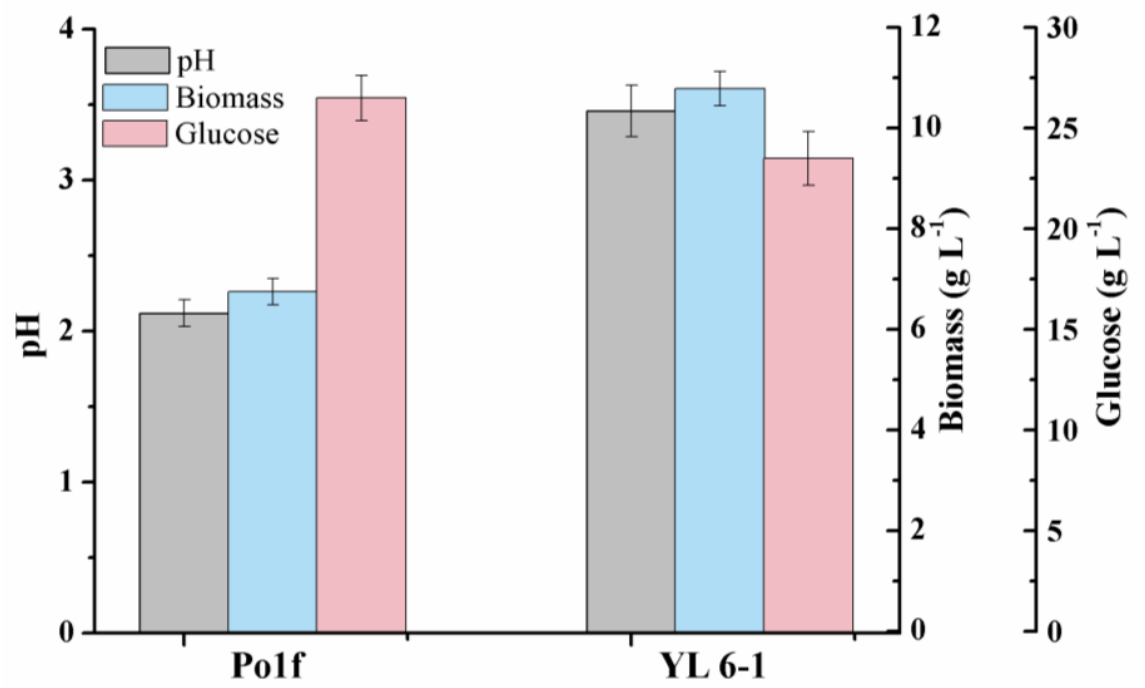

Fig. 4 